\title{
EXPLICIT EFFICIENT FRONTIER OF A CONTINUOUS-TIME MEAN-VARIANCE PORTFOLIO SELECTION PROBLEM
}

\author{
Xun Yu Zhou* and Duan $\mathrm{Li}^{\dagger}$ \\ Department of Systems Engineering and Engineering Management \\ The Chinese University of Hong Kong \\ Shatin, NT \\ Hong Kong
}

xyzhou@se.cuhk.edu.hk and dli@se.cuhk.edu.hk

\begin{abstract}
In this work we consider a continuous-time mean-variance portfolio selection problem that is formulated as a bi-criteria optimization problem. The objective is to maximize the expected return and minimize the variance of the terminal wealth. By putting weights on the two criteria one obtains a single objective stochastic control problem which is however not in the standard form. We show that this non-standard problem can be "embedded" into a class of auxiliary stochastic linear-quadratic (LQ) problems. By solving the latter based on the recent development on stochastic LQ problems with indefinite control weighting matrices, we derive the efficient frontier in a closed form for the original mean-variance problem.
\end{abstract}

\section{INTRODUCTION}

Portfolio selection is to seek a best allocation of wealth among a basket of securities. The mean-variance approach by Markowitz [9] provides a fundamental basis for portfolio construction in a single period. The most important contribution of this model is that it quantifies the risk by using the variance which enables investors to seek highest return after specifying their acceptable risk level. This approach becomes the foundation of modern finance theory and inspires literally hundreds of extensions and applications. In particular, in the case where the variance matrix is positive definite and short-selling is allowed, an analytic solution was obtained by Merton [10]. Perold [12] developed a more

*The research of this author was supported by RGC Earmarked Grant CUHK 4125/97E.

${ }^{\dagger}$ The research of this author was supported by RGC Earmarked Grant CUHK 4130/97E.

The original version of this chapter was revised: The copyright line was incorrect. This has been corrected. The Erratum to this chapter is available at DOI: 10.1007/978-0-387-35359-3_40 
general technique to locate the efficient frontier when the covariance matrix is nonnegative definite.

After Markowitz's pioneering work, the mean-variance model was soon extended to multi-period portfolio selection, see for example Mossin [11], Samuelson [13], Elton and Gruber [5], and Grauer and Hakansson [7]. The meanvariance model in a continuous-time setting has been developed a bit later, see Föllmer-Sondermann [6], Duffie and Jackson [3], and Duffie and Richardson [4]. The basic approach in these works is the dynamic programming.

The purpose of this paper is to seek optimal portfolio policy for dynamic investment problems with a mean-variance formulation in continuous-time. Different from the approach used in Duffie and Richardson [4], we employ the embedding technique, introduced by $\mathrm{Li}$ and $\mathrm{Ng}$ [8] for the multi-period, discretetime portfolio selection problem, which leads to a stochastic linear-quadratic control problem (LQ problem, for short), and then apply the stochastic LQ theory recently developed by Chen, Li and Zhou [1] and Chen and Zhou [2] to solve the problem.

This paper is an announcement of the major results in Zhou and $\mathrm{Li}$ [15], where the detailed proofs are presented.

\section{PROBLEM FORMULATION}

Suppose there is a market in which $m+1$ assets (or securities) are traded continuously. One of the assets is the bond whose price process $P_{0}(t)$ is subject to the following (deterministic) ordinary differential equation:

$$
\left\{\begin{array}{l}
d P_{0}(t)=r(t) P_{0}(t) d t, t \in[0, T] \\
P_{0}(0)=p_{0}>0
\end{array}\right.
$$

where $r(t)>0$ is called the interest rate (of the bond). The other $m$ assets are called stocks whose price processes $P_{1}(t), \cdots, P_{m}(t)$ satisfy the following stochastic differential equation:

$$
\left\{\begin{array}{l}
d P_{i}(t)=P_{i}(t)\left\{b_{i}(t) d t+\sum_{j=1}^{m} \sigma_{i j}(t) d W_{j}(t)\right\}, t \in[0, T] \\
P_{i}(0)=p_{i}>0
\end{array}\right.
$$

where $b_{i}(t)>0$ is called the appreciation rate, and $\sigma_{i}(t) \equiv\left(\sigma_{i 1}(t), \cdots, \sigma_{i m}(t)\right)$ : $[0, T] \times \Omega \rightarrow R^{m}$ is called the volatility or the dispersion of the stocks. Here,

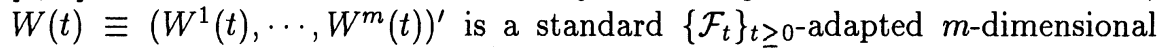
Brownian motion defined on some fixed filtered complete probability space $\left(\Omega, \mathcal{F}, P,\left\{\mathcal{F}_{t}\right\}_{t \geq 0}\right)$. Define the covariance matrix

$$
\sigma(t)=\left(\begin{array}{l}
\sigma_{1}(t) \\
\vdots \\
\sigma_{m}(t)
\end{array}\right) \equiv\left(\sigma_{i j}(t)\right)_{m \times m}
$$

The basic assumption throughout this paper is

$$
\sigma(t) \sigma(t)^{\prime} \geq \delta I, \quad \forall t \in[0, T]
$$


for some $\delta>0$. This is the so-called non-degeneracy assumption. We also assume that all the functions are measurable and uniformly bounded in $t$.

Consider an investor whose total wealth at time $t \geq 0$ is denoted by $x(t)$. Suppose he/she decides to hold $N_{i}(t)$ shares of $i$-th asset $(i=0,1, \cdots, m)$ at time $t$. Then

$$
x(t)=\sum_{i=0}^{m} N_{i}(t) P_{i}(t), \quad t \geq 0 .
$$

Assume that the trading of shares takes place continuously and there is no transaction cost and consumptions during the whole time period $[0, T]$. Then one has

$$
\left\{\begin{aligned}
d x(t)= & \sum_{i=0}^{m} N_{i}(t) d P_{i}(t) \\
= & \left\{r(t) N_{0}(t) P_{0}(t)+\sum_{i=1}^{m} b_{i}(t) N_{i}(t) P_{i}(t)\right\} d t \\
& +\sum_{i=1}^{m} N_{i}(t) P_{i}(t) \sum_{j=1}^{m} \sigma_{i j}(t) d W_{j}(t) \\
= & \left\{r(t) x(t)+\sum_{i=1}^{m}\left[b_{i}(t)-r(t)\right] u_{i}(t)\right\} d t \\
& +\sum_{j=1}^{m} \sum_{i=1}^{m} \sigma_{i j}(t) u_{i}(t) d W_{j}(t), \\
x(0)= & x_{0}>0,
\end{aligned}\right.
$$

where

$$
u_{i}(t) \equiv N_{i}(t) P_{i}(t), \quad i=0,1,2 \cdots, m,
$$

denotes the total market value of the investor's wealth in the $i$-th bond/stock. If $u_{i}(t)<0(i=1,2, \cdots, m)$, then the investor is short-selling $i$-th stock. If $u_{0}(t)<0$, then the investor is borrowing the amount $\left|u_{0}(t)\right|$ at rate $r(t)$. It is clear that by changing $u_{i}(t)$, the investor changes the "allocation" of his wealth in these $m+1$ assets. We call $u(t)=\left(u_{1}(t), \cdots, u_{m}(t)\right)^{\prime}$ a portfolio of the investor. Notice that we exclude the allocation to the bond, $u_{0}(t)$, from the portfolio as it will be completely determined by the allocations to the stocks. The objective of the investor is to maximize the mean terminal wealth, $\operatorname{Ex}(T)$, and at the same time to minimize the variance of the terminal wealth

$$
\operatorname{Var} x(T) \equiv E[x(T)-E x(T)]^{2}=E x(T)^{2}-[E x(T)]^{2} .
$$

This is a multi-objective optimization problem with two criteria in conflict.

Let us denote by $L_{\mathcal{F}}^{2}\left(0, T ; R^{m}\right)$ the set of all $R^{m}$-valued, measurable stochastic processes $f(t)$ adapted to $\left\{\mathcal{F}_{t}\right\}_{t \geq 0}$, such that $E \int_{0}^{T}|f(t)|^{2} d t<\infty$.

Definition 1. A portfolio $u(\cdot)$ is said to be admissible if $u(\cdot) \in L_{\mathcal{F}}^{2}\left(0, T ; R^{m}\right)$.

Definition 2. The mean-variance portfolio optimization problem is denoted as

$$
\begin{gathered}
\text { Minimize } \\
\text { Subject to }
\end{gathered}\left\{\begin{array}{l}
\left.J_{1}(u(\cdot)), J_{2}(u(\cdot))\right) \equiv(-E x(T), \operatorname{Var} x(T)), \\
u(\cdot) \in L_{\mathcal{F}}^{2}\left(0, T ; R^{m}\right), \\
(x(\cdot), u(\cdot)) \text { satisfy equation }(6) .
\end{array}\right.
$$

Moreover, an admissible portfolio $\bar{u}(\cdot)$ is called an efficient portfolio of the problem if there exists no admissible portfolio $u(\cdot)$ such that

$$
J_{1}(u(\cdot)) \leq J_{1}(\bar{u}(\cdot)), \quad J_{2}(u(\cdot)) \leq J_{2}(\bar{u}(\cdot))
$$


and at least one of the inequalities holds strictly. In this case, $\left(J_{1}(\bar{u}(\cdot)), J_{2}(\bar{u}(\cdot))\right)$ $\in R^{2}$ is called an efficient point. The set of all efficient points is called the efficient frontier.

In other words, an efficient portfolio is one that there exists no other portfolio better than it with respect to both the mean and variance criteria. The problem then is to identify the efficient portfolios along with the efficient frontier. By standard multi-objective optimization theory (see Zeleny [14]), an efficient portfolio can be found under certain convexity condition by solving a singleobjective optimization problem where the objective is a weighted average of the two original criteria. The efficient frontier can then be generated by varying the weights. Therefore, the original problem can be solved via the following optimal control problem

$$
\begin{array}{cl}
\text { Minimize } & J_{1}(u(\cdot))+\mu J_{2}(u(\cdot)) \equiv-E x(T)+\mu \operatorname{Var} x(T), \\
\text { Subject to } & \left\{\begin{array}{l}
u(\cdot) \in L_{\mathcal{F}}^{2}\left(0, T ; R^{m}\right), \\
(x(\cdot), u(\cdot)) \text { satisfy equation }(6),
\end{array}\right.
\end{array}
$$

where the parameter (representing the weight) $\mu>0$. Denote the above problem by $P(\mu)$. Define

$$
\Pi_{P(\mu)}=\{u(\cdot) \mid u(\cdot) \text { is an optimal control of } P(\mu)\} .
$$

\section{CONSTRUCTION OF AUXILIARY PROBLEM}

Note that Problem $P(\mu)$ is not a standard stochastic optimal control problem and is hard to solve directly due to the term $[E x(T)]^{2}$ in its cost function which is non-separable in the sense of dynamic programming. We now propose to embed the problem into a tractable auxiliary problem that turns out to be a stochastic linear-quadratic (LQ) problem. To do this, set

$$
\begin{array}{cl}
\text { Minimize } & J(u(\cdot) ; \mu, \lambda) \equiv E\left\{\mu x(T)^{2}-\lambda x(T)\right\}, \\
\text { Subject to } & \left\{\begin{array}{l}
u(\cdot) \in L_{\mathcal{F}}^{2}\left(0, T ; R^{m}\right), \\
(x(\cdot), u(\cdot)) \text { satisfy equation (6), }
\end{array}\right.
\end{array}
$$

where the parameters $\mu>0$ and $-\infty<\lambda<+\infty$. Let us call the above Problem $A(\mu, \lambda)$. Define

$$
\Pi_{A(\mu, \lambda)}=\{u(\cdot) \mid u(\cdot) \text { is an optimal control of } A(\mu, \lambda)\} .
$$

The following result tells the relationship between the problems $P(\mu)$ and $A(\mu, \lambda)$.

Theorem 1. For any $\mu>0$, one has

$$
\Pi_{P(\mu)} \subseteq \bigcup_{-\infty<\lambda<+\infty} \Pi_{A(\mu, \lambda)}
$$


Moreover, if $\bar{u}(\cdot) \in \Pi_{P(\mu)}$, then $\bar{u}(\cdot) \in \Pi_{A(\mu, \bar{\lambda})}$ with $\bar{\lambda}=1+2 \mu E \bar{x}(T)$.

The implication of Theorem 1 is that any optimal solution of the problem $P(\mu)$ (as long as it exists) can be found via solving Problem $A(\mu, \lambda)$. Notice that the auxiliary problem $A(\mu, \lambda)$ is a standard stochastic optimal control problem (parameterized by $(\mu, \lambda)$ ), separable in the sense of dynamic programming and of a linear-quadratic structure.

\section{SOLUTION TO AUXILIARY PROBLEM}

Now let us solve Problem $A(\mu, \lambda)$. Putting

$$
\gamma=\frac{\lambda}{2 \mu} \text { and } y(t)=x(t)-\gamma
$$

Problem $A(\mu, \lambda)$ is equivalent to minimizing

$$
E\left[\frac{1}{2} \mu y(T)^{2}\right]
$$

subject to

$$
\left\{\begin{aligned}
d y(t)= & \{A(t) y(t)+B(t) u(t)+b(t)\} d t \\
& +\sum_{j=1}^{m} D_{j}(t) u(t) d W^{j}(t), \\
y(0)= & x_{0}-\gamma
\end{aligned}\right.
$$

where

$$
\begin{cases}A(t)=r(t), & B(t)=\left(b_{1}(t)-r(t), \cdots, b_{m}(t)-r(t)\right) \\ b(t)=\gamma r(t), & D_{j}(t)=\left(\sigma_{1 j}(t), \cdots, \sigma_{m j}(t)\right)\end{cases}
$$

This is a typical stochastic LQ control problem as the dynamics (18) of this problem is linear (with a nonhomogeneous term in the drift) and the cost (17) is quadratic. Moreover, the running cost (over the period $[0, T]$ ) in (17) is absent, so it is a problem with indefinite control weighting running cost, one that was recently extensively investigated by Chen, Li and Zhou [1], and Chen and Zhou [2]. Applying the general results in $[1,2]$ and making use of the fact that the state variable $x(t)$ in the present case is scalar-valued, we get the following result.

Theorem 2. The optimal state feedback control for the problem $A(\mu, \lambda)$ is given by

$$
\begin{aligned}
\bar{u}(t, x) & \equiv\left(\bar{u}_{1}(t, x), \cdots, \bar{u}_{m}(t, x)\right) \\
& =\left(\sigma(t) \sigma(t)^{\prime}\right)^{-1} B(t)^{\prime}\left(\gamma e^{-\int_{t}^{T} r(s) d s}-x\right)
\end{aligned}
$$




\section{EFFICIENT FRONTIER}

Under the optimal feedback control (20), the wealth equation (6) evolves as

$$
\left\{\begin{aligned}
d x(t)= & \left\{(r(t)-\rho(t)) x(t)+\gamma e^{-\int_{t}^{T} r(s) d s} \rho(t)\right\} d t \\
& +B(t)\left(\sigma(t) \sigma(t)^{\prime}\right)^{-1} \sigma(t)\left(\gamma e^{-\int_{t}^{T} r(s) d s}-x(t)\right) d W(t) \\
x(0)= & x_{0},
\end{aligned}\right.
$$

where

$$
\rho(t)=B(t)\left(\sigma(t) \sigma(t)^{\prime}\right)^{-1} B(t)^{\prime} .
$$

Moreover, applying Ito's formula to $x(t)^{2}$, we obtain

$$
\left\{\begin{array}{l}
d x(t)^{2}=\left\{(2 r(t)-\rho(t)) x(t)^{2}+\gamma^{2} e^{-2 \int_{t}^{T} r(s) d s} \rho(t)\right\} d t \\
+2 x(t) B(t)\left(\sigma(t) \sigma(t)^{\prime}\right)^{-1} \sigma(t)\left(\gamma e^{-\int_{t}^{T} r(s) d s}-x(t)\right) d W(t) \\
x(0)^{2}=x_{0}^{2}
\end{array}\right.
$$

Taking expectations on both sides of (21) and (22), we conclude that $E x(t)$ and $E x(t)^{2}$ satisfy the following two ordinary differential equation:

$$
\left\{\begin{aligned}
d E x(t) & =\left\{(r(t)-\rho(t)) E x(t)+\gamma e^{-\int_{t}^{T} r(s) d s} \rho(t)\right\} d t \\
E x(0) & =x_{0}
\end{aligned}\right.
$$

and

$$
\left\{\begin{aligned}
d E x(t)^{2} & =\left\{(2 r(t)-\rho(t)) E x(t)^{2}+\gamma^{2} e^{-2 \int_{t}^{T} r(s) d s} \rho(t)\right\} d t \\
E x(0)^{2} & =x_{0}^{2}
\end{aligned}\right.
$$

Solving (23) and (24), we can express $E x(T)$ and $E x(T)^{2}$ as explicit functions of $\gamma$,

$$
E x(T)=\alpha x_{0}+\beta \gamma, \quad E x(T)^{2}=\delta x_{0}^{2}+\beta \gamma^{2},
$$

where

$$
\alpha=e^{\int_{0}^{T}(r(t)-\rho(t)) d t}, \quad \beta=1-e^{-\int_{0}^{T} \rho(t) d t}, \quad \delta=e^{\int_{0}^{T}(2 r(t)-\rho(t)) d t} .
$$

By Theorem 1, an optimal solution of the problem $P(\mu)$, if it exists, can be found by selecting $\bar{\lambda}$ so that

$$
\bar{\lambda}=1+2 \mu E \bar{x}(T)=1+2 \mu\left(\alpha x_{0}+\beta \frac{\bar{\lambda}}{2 \mu}\right) .
$$

This yields

$$
\bar{\lambda}=\frac{1+2 \mu \alpha x_{0}}{1-\beta}=e^{\int_{0}^{T} \rho(t) d t}+2 \mu x_{0} e^{\int_{0}^{T} r(t) d t} .
$$


Hence the optimal control for the problem $P(\mu)$ is given by (20) with $\gamma=\bar{\gamma}=$ $\frac{\bar{\lambda}}{2 \mu}$ and $\bar{\lambda}$ given by (27). Therefore one can calculate the corresponding variance of the terminal wealth in terms of the expectation. This leads to the efficient frontier we are seeking.

Theorem 3. The efficient frontier of the bi-criteria optimal portfolio selection problem (9), if it ever exists, must be given by the following

$$
\operatorname{Var} \bar{x}(T)=\frac{e^{-\int_{0}^{T} \rho(t) d t}}{1-e^{-\int_{0}^{T} \rho(t) d t}}\left(E \bar{x}(T)-x_{0} e^{\int_{0}^{T} r(t) d t}\right)^{2} .
$$

The relationship (28) reveals explicitly the trade-off between the mean (return) and variance (risk). For example, if one has set an expected return level, then the above can tell the risk he/she has to take; and vice versa. In particular, if one cannot take any risk, namely, $\operatorname{Var}(\bar{x}(T))=0$, then $E \bar{x}(T)$ has to be $x_{0} e^{\int_{0}^{T} r(t) d t}$ meaning that he/she can only put his/her money in the bond. Another interesting phenomenon is that the efficient frontier (28) involves a perfect square. This is due to the possible inclusion of the bond in a portfolio. In the case when the riskless bond is excluded from consideration, then the efficient frontier may no longer be a perfect square, which means one cannot have a risk-free portfolio.

\section{References}

[1] Chen, S., Li, X., and Zhou, X. Y., Stochastic linear quadratic regulators with indefinite control weight costs, SIAM J. Control E Optim., 36 (1998), 1685-1702.

[2] Chen, S. and Zhou, X. Y., Stochastic linear quadratic regulators with indefinite control weight costs, II, preprint.

[3] Duffie, D. and Jackson, M., Optimal hedging and equilibrium in a dynamic futures market, J. Econ. Dynam. Control., 14 (1990), 21-33.

[4] Duffie, D. and Richardson, H., Mean-variance hedging in continuous time, Ann. Appl. Probab., 1 (1991), 1-15.

[5] Elton, E. J. and Gruber M. J., Finance as a Dynamic Process, Prentice Hall, Englewood Cliffs, 1975.

[6] Föllmer, H. and Sondermann, D., Hedging of non-redundant contingent claims, in Contributions to Mathematical Economics, A. Mas-Colell and W. Hildenbrand ed., North-Holland, Amsterdam, 1986, 205-223.

[7] Grauer, R. R. and Hakansson, N. H., On the use of mean-variance and quadratic approximations in implementing dynamic investment strategies: a comparison of returns and investment policies, Manag. Sci., 39 (1993), 856-871.

[8] Li, D. and Ng, W. L., Optimal dynamic portfolio selection : Multi-period mean-variance formulation, submitted for publication, 1997. 
[9] Markowitz, H., Portfolio selection, J. of Fin., 7 (1952), 77-91.

[10] Merton, R. C., An analytic derivation of the efficient portfolio frontier, $J$. Fin. Quant. Anal., 7 (1972), 1851-1872.

[11] Mossin J., Optimal multiperiod portfolio policies, J. of Business, 41 (1968), 215-229.

[12] Perold, A.d F., Large-scale portfolio optimization, Manag. Sci., 30 (1984), 1143-1160.

[13] Samuelson, P. A., Lifetime portfolio selection by dynamic stochastic programming, Rev. of Econ. and Stat., 51 (1969), 239-246.

[14] Zeleny, M., Multiple Criteria Decision Making, McGraw-Hill, New York, 1981.

[15] Zhou, X. Y. and Li, D., Continuous-time mean-variance formulation in dynamic portfolio selection, preprint. 